5 Bebbington $\mathrm{P}$, Jonas $\mathrm{S}$, Kuipers $\mathrm{E}$, King $\mathrm{M}$, Cooper $\mathrm{C}$, Brugha $\mathrm{T}$, et al. Childhood sexual abuse and psychosis: data from a cross-sectional nationa psychiatric survey in England. Br J Psychiatry 2011; 199: 29-37.

6 Varese F, Smeets F, Drukker M, Lieverse R, Lataster T, Viechtbauer W, et al. Childhood adversities increase the risk of psychosis: a meta-analysis of patient-control, prospective- and cross-sectional cohort studies. Schizophr Bull 2012; 38: 661-71.

Paul E. Bebbington, Emeritus Professor, Division of Psychiatry, University College London, London, UK. Email: p.bebbington@ucl.ac.uk

doi: 10.1192/bjp.205.1.77a

Authors' reply: We thank Professor Bebbington for his comments and for giving us the opportunity to clarify our recommendations relating to copy number variant $(\mathrm{CNV})$ testing in those with schizophrenia. We would like to make it clear that we were not suggesting universal screening of CNVs in healthy populations. Rather, we were suggesting that it is time to consider testing for CNVs in those with a diagnosis of schizophrenia. On reflection, we should have used the term 'genetic testing' rather than 'screening', and apologise for this ambiguity. In this sense the positive predictive value of CNVs for schizophrenia is irrelevant as the patient already has the disorder.

We believe that testing for pathogenic CNVs in schizophrenia should be considered for a number of reasons, but emphasise that this should only be undertaken with clear informed consent and in the context of professional genetic counselling. Among the potential benefits of knowing the carrier status of patients, physical health and informing patients about potential risks to family/offspring are the two areas that stand out.

Therefore, if we diagnose a patient with schizophrenia as a carrier of a pathogenic CNV, even though this will apply to only $2-3 \%$ of our patients, it could have important implications for their management. The identified CNVs can have an adverse impact on patients' health given that these CNVs are associated with obesity, epilepsy and cardiovascular disorders. This information could be crucial in guiding targeted monitoring and intervention, particularly given the increasing recognition of the effects of poor physical health and decreased life expectancy in schizophrenia. These factors may also be important considerations in the selection of the most appropriate medication.

Further, although the frequency of the implicated CNVs is low in schizophrenia, each of the 11 implicated CNVs can lead to a range of other disorders such as developmental delay, intellectual deficit, autism spectrum disorders, and a number of congenital anomalies. We have estimated that carriers of these CNVs have substantial risk of developing one of these serious disorders. ${ }^{1}$ The risk ranges from $10.6 \%$ for the duplication at $16 \mathrm{p} 13.11$ to nearly $100 \%$ for the deletion at 22q11.2, with an average of $42.8 \%$. The penetrance solely for schizophrenia is indeed relatively low, ranging from 2 to $12 \%$ (assuming a $1 \%$ lifetime risk for schizophrenia). Taken together, we feel that this information could be helpful to patients in making decisions about having children and potentially for their wider family.

There is currently a lack of research into the possible benefits and risks of such genetic testing and we would strongly advocate for such research before the implementation of CNV testing programmes. This should be informed by the wealth of experience in genetic counselling that has developed in other genetic disorders. We feel many patients and families would find this information helpful in rationalising a cause for the illness and that this may help lessen the guilt experienced by many families. We appreciate the chance to have begun this debate and would stress that the views of patients with schizophrenia and their families relating to genetic testing should be central to the debate and future research.
1 Kirov G, Rees E, Walters JT, Escott-Price V, Georgieva L, Richards AL, et al. The penetrance of copy number variations for schizophrenia and developmental delay. Biol Psychiatry 2014; 75: 378-85.

Elliott Rees, MRes, James T. R. Walters, PhD, MRCPsych, Michael J. Owen, PhD, FRCPsych, George Kirov, PhD, MRCPsych, Centre for Neuropsychiatric Genetics and Genomics, Institute of Psychological Medicine and Clinical Neurosciences, Cardiff University, Cardiff, UK. Email: kirov@cardiff.ac.uk

doi: 10.1192/bjp.205.1.78

\section{Getting real about risk}

The recent meta-analysis by Singh et $a l^{1}$ examined the proportion of violent people among those classified as high risk, known as the positive predictive value (PPV). They found that PPV is highly variable between studies and is most strongly associated with the base rate of violence in the whole risk-assessed group. They conclude that risk assessment is not a reliable indicator of absolute risk. We agree. The increased focus on the PPV of high-risk categories is a welcome development because it leads to a consideration of the number of people who might need to be assessed as high-risk for every true positive (actually violent) person. The number needed to assess is often a more relevant measure than those derived from the receiver operator curve and it clearly illustrates the lack of meaning in recent debates about the extent to which group data apply to individuals ${ }^{2}$ and the margins of error in particular risk predictions. ${ }^{3}$ However, we believe that the debate about risk assessment now needs to move beyond abstract notions relating solely to probability. A probability after all is simply a number between 0 and 1 , a number that is uninformative unless it is a probability of something specific.

Although not cited in Singh et al, we systematically examined PPV of risk categorisation after making generous assumptions about the statistical power of risk assessment. ${ }^{4}$ Unlike Singh et al, our paper focused on the main factor that actually determines base rates and thus PPV - the definition of violence according to violence severity. For example, using a risk assessment instrument with a sensitivity and specificity of $80 \%$ for the detection of different outcomes, the PPV for criminally violent behaviour over a year by people with schizophrenia might be about $4 \%$ under optimal conditions, whereas the same figure for homicide would be $0.04 \%$.

In the primary risk research, including that used by Singh et $a l,{ }^{1}$ a wide spectrum of violent events is amalgamated into an omnibus 'violent' category. These events range from common assault all the way to homicide. Each of these diverse events has different base rates and consequences, with more severe violence having lower base rate but leading to greater losses.

Risk assessment in mental health should start to consider the dimension of resulting loss. In areas outside mental health, risk is not a probability but is a quantum of loss - that is why we pay our insurance premium in money, yet have little idea of the likelihood of the loss of our possessions. In our view any study that does not consider the magnitude of resulting loss should not really be thought of as a 'risk assessment' and more properly should be referred to as 'probability assessment'. Although quantification of loss poses significant challenges, considering a definition of risk that includes the loss component re-emphasises two complex, important and unanswered questions. First, what actual psychiatric interventions in terms of cost/side-effects/benefits are indicated for those who are regarded as high risk, and yet should be withheld from patients classified as low risk? If the harm we consider is not severe, no costly, restrictive or intrusive treatment can be justified. If the harm considered is severe, it will also be rare. Therefore, what costly and intrusive intervention can be justified for the tiny proportion of false positives, or if the intervention is not costly or intrusive, why withhold it from 
low-risk patients, who will commit many of the future acts of violence? $?^{5}$ Second, is there evidence that an overall reduction in violence can be achieved by applying this cost-effective and acceptable intervention to a group who are more likely to offend while denying it to those who as a group are less likely to offend? Will the additional resources spent on preventing violence by high-risk patients be justified in terms of harm reduction? ${ }^{6}$

At the end of the recent paper, Singh et al recommend that risk assessments be provided with a qualification explaining their limitations. Here we agree as well. Perhaps it should be 'this risk assessment provides an estimate of an uncertain probability of an unspecified event with no consideration of the consequences'.

1 Singh JP, Fazel S, Gueorguieva R, Buchanan A. Rates of violence in patients classified as high risk by structured risk assessment instruments. Br J Psychiatry 2014; 204: 180-7.

2 Scurich N, Monahan J, John RS. Innumeracy and unpacking: bridging the nomothetic/idiographic divide in violence risk assessment. Law Hum Behav 2012; 36: 548-54

3 Hart SD, Michie C, Cooke DJ. Precision of actuarial risk assessment instruments: evaluating the 'margins of error' of group $v$. individual predictions of violence. Br J Psychiatry 2007; 190 (suppl 49): s60-5.

4 Large MM, Ryan CJ, Singh SP, Paton MB, Nielssen OB. The predictive value of risk categorization in schizophrenia. Harv Rev Psychiatry 2011; 19: 25-33.

5 Large M, Ryan CJ. Screening for suicide: a comment on Steeg et al. Psychol Med 2012; 42: 2011-2; author reply 2-3.

6 Wand $\mathrm{T}$, Large $\mathrm{M}$. Little evidence for the usefulness of violence risk assessment. Br J Psychiatry 2013; 202: 468.

Matthew Large, Psychiatrist, School of Psychiatry, University of New South Wales, Kensington, Australia. Email: mmbl@bigpond.com; Swaran P. Singh, Professor and Head of Division, Mental Health and Wellbeing, Warwick Medical School, University Head of Division, Mental Heak
of Warwick, Coventry, UK.

doi: 10.1192/bjp.205.1.78a
Authors' reply: We thank Large \& Singh for their comments. But we would point out that we did not examine positive predictive value, as they say we did. We described the proportion of those classified as high risk who then acted violently. The two are only the same if an ascription of high risk, whether made using a structured risk assessment instrument (SRAI) or arrived at through clinical judgement, is treated as a 'prediction'. Studies of the predictive validity of risk instruments out of necessity handle the data in this way ${ }^{1}$ and usually conclude that SRAIs demonstrate a moderate level of accuracy. As those who design SRAIs and others have repeatedly pointed out, however, fallible predictions are of limited value to clinicians. ${ }^{2}$ One thing that should help those clinicians is knowing what a classification of high risk means and, in particular, whether it means the same thing in different settings. We found that after controlling for time at risk, the rate of violence in groups classified as high risk using SRAIs shows substantial variation.

1 Fazel S, Singh JP, Doll H, Grann M. The prediction of violence and antisocial behaviour: a systematic review and meta-analysis of the utility of risk assessment instruments in 73 samples involving 24,827 individuals. BMJ 2012; 345: e4692.

2 Otto R, Douglas K. Handbook of Violence Risk Assessment. Routledge, 2010.

Alec Buchanan, PhD, MD, Department of Psychiatry, Yale University, New Haven, Connecticut, USA. Email: alec.buchanan@yale.edu; Seena Fazel, MD, Department of Psychiatry, University of Oxford, Warneford Hospital, Oxford, UK.

doi: 10.1192/bjp.205.1.79 